\title{
CONDICIONES TROPICALES DE LA LLUVIA ESTACIONAL EN LA AGRICULTURA DE SECANO DE CARABOBO, VENEZUELA
}

\author{
TROPICAL RAINFALL CONDITIONS IN RAINFED AGRICULTURE IN CARABOBO, \\ VENEZUELA
}

\section{Barlin Orlando Olivares}

Investigador. Programa Iberoamericano de Doctores en Agroalimentación de la Universidad de Córdoba (UCO), Andalucía, España.

*Autor para correspondencia: barlinolivares@gmail.com

\begin{abstract}
Resumen
La variabilidad y el cambio climático están teniendo fuertes repercusiones sobre diferentes variables meteorológicas; induciendo cambios en el patrón de precipitación que afecta directamente la disponibilidad hídrica en los agroecosistemas. Es por esta razón que el objetivo de este trabajo fue analizar la variabilidad interanual de la lluvia tropical y su influencia en la agricultura de secano de la cuenca del Lago de Valencia en Venezuela. Para esto se utilizaron los datos mensuales y anuales de precipitación (1969-1999) de ocho estaciones climatológicas. Posteriormente, se calcularon los estadísticos descriptivos básicos, se describió la evolución de la precipitación y por último, se calculó del rango percentil $\mathrm{P} 90 \%-\mathrm{P} 10 \%$ como indicador de la variación de la amplitud de la serie. Los resultados establecen que existen cambios en el régimen interanual de la precipitación y, tras analizar las tendencias en la precipitación anual en las localidades Yuma Caserío, Colonia El Trompillo, Agua Blanca y Las Dos Bocas, existe reducción generalizada en la lluvia, estos cambios, afectarían negativamente la generación y la disponibilidad de humedad en las localidades agrícolas, las cuales dependen fuertemente del agua de lluvia. La vocación agrícola en las tierras bajas de la cuenca del lago de Valencia, las hacen muy vulnerables a los episodios secos persistentes, donde los cultivos de secano como sorgo industrial, maíz, hortalizas, frutas (musáceas) y cítricos merman sus rendimientos durante la ausencia prolongada de lluvias, lo que afecta al sector agrícola de la región.
\end{abstract}

Palabras claves: Estacionalidad, precipitación, tendencia, agrometeorología. 


\begin{abstract}
Variability and climate change are having a strong impact on different meteorological variables; inducing changes in the precipitation pattern that directly affects water availability in agroecosystems. It is for this reason that the objective of this work was to analyze the interannual variability of tropical rainfall and its influence on rainfed agriculture of the Lake of Valencia basin in Venezuela. For this, the monthly and annual precipitation data (1969-1999) of eight climatological stations were used. Subsequently, the basic descriptive statistics were calculated, the evolution of the precipitation was described and finally, it was calculated from the $\mathrm{P} 90 \%-10 \%$ percentile range as an indicator of the variation of the series amplitude. The results establish that there are changes in the interannual precipitation regime and, after analyzing trends in annual precipitation in Yuma Caserio, Colonia El Trompillo, Agua Blanca and Las Dos Bocas, there is a generalized reduction in rainfall, would negatively affect the generation and availability of moisture in agricultural locations, which depend heavily on rainwater. The agricultural vocation in the lowlands of the lake basin of Valencia makes it very vulnerable to persistent dry episodes, where rainfed crops such as industrial sorghum, maize, vegetables, fruits (musáceas) and citrus fruits diminish their yield during the absence prolonged rainfall, affecting the agricultural sector of the region.
\end{abstract}

Keywords: Seasonality, precipitation, tendency, agrometeorology.

Forma sugerida de citar: Olivares, B. O. 2018. Condiciones tropicales de la lluvia estacional en la agricultura de secano de Carabobo, Venezuela. La Granja: Revista de Ciencias de la Vida. Vol. 27(1):86-102. http: //doi.org/10.17163/lgr.n27.2018.07. 


\section{Introducción}

Para la subsistencia del sector agrícola, que se enfrenta a crecientes riesgos climáticos, es fundamental la información oportuna y precisa sobre el tiempo, el clima y el agua. Las observaciones, las predicciones y los análisis realizados en investigaciones permiten a las comunidades agrícolas aumentar la producción pecuaria y el rendimiento de cultivos, planificar las temporadas de siembra y cosecha, y reducir las plagas o enfermedades (OMM, 2009; Olivares, 2009).

La agricultura tropical de secano desarrollada en el país, y específicamente la del estado Carabobo, es considerada como un negocio arriesgado, fundamentalmente por la influencia de la variabilidad intranual e interanual en la disponibilidad de agua de los cultivos y sobre la posibilidad de realizar labores agrícolas mecanizadas.

Las características tropicales de las zonas subhúmedas bajo estudio, están representadas por la precipitación superior a la evapotranspiración en parte del año e inferior en otros meses (déficit estacional más común y severo, el régimen de precipitación puede ser muy errático); cuyo ambiente dominante está caracterizado por presentar suelos de alta a moderada fertilidad, en donde se encuentra gran parte de la producción de hortalizas, maíz y caña de azúcar de la región central. Predominan las tierras de clase (I), con suelos profundos bien drenados y fáciles de trabajar, sin ninguna o ligeras restricciones para su uso (Comerma y Parede, 1978; Zinck, 2012).

Según las investigaciones desarrolladas por Martelo (2003); Guenni, Degryze y Alvarado (2008); Guevara, Guevara y García (2008); Lobo et al. (2010); Cortez et al. (2011); Olivares et al. (2012); Olivares, Caraballo y Torrealba (2013); Paredes et al. (2015), se establece que a mediados del mes de abril el desplazamiento al norte del anticiclón del Atlántico, permite el avance de la Zona de Convergencia Intertropical (ZCIT), estableciéndose sobre gran parte del país. La ZCIT permite señalar el inicio de la temporada lluviosa en Venezuela la cual se extiende hasta principios de noviembre. Con el retiro progresivo de la ZCIT al sur; fuera del territorio venezolano, y el aumento de la influencia del anticiclón del Atlántico sobre el país, se establece el inicio de la temporada seca, la cual se extiende hasta el mes de abril.

La lluvia es considerada como un proceso espacio-temporal dentro del sistema climático, ya que exhibe aspectos aleatorios tanto en el tiempo como en el espacio y cuya estructura de variabilidad pueden explorarse mediante técnicas estadísticas apropiadas (Pradere, 1999; Toledo y HernándezSzczurek, 2003). Uno de los aspectos a considerar en los estudios de meteorología lo representa la evolución de la precipitación y el posible impacto que la variabilidad climática tenga en la misma, es por ello que la variabilidad de la lluvia tropical estacional reviste importancia como parámetro descriptivo de las series de observación y más cuando tiene implicaciones en la agricultura de secano.

El objetivo de esta investigación es estudiar y definir las principales características de los eventos de lluvia tropical estacional de importancia en la agricultura de secano, desarrollada en la cuenca del Lago de Valencia, estado Carabobo, Venezuela. En este sentido, la iniciativa de desarrollar investigaciones sobre la evolución probable del clima, la variabilidad de los elementos climáticos y la influencia en las actividades socioeconómicas, contribuyen a mejorar la producción y la sostenibilidad agrícola y, al mismo tiempo, la seguridad alimentaria de la nación.

\section{Materiales y métodos}

\section{1 Área de estudio}

En la zona de estudio, la región natural predominante es la depresión del Lago de Valencia, comprendiendo la Serranía del Litoral, del Interior y la Depresión de Valencia, la cual constituye tipos de paisajes de montañas y valles, con una superficie de $2300 \mathrm{~km}^{2}$ (MARN, 2005). Los valles que bordean el lago y el resto de paisajes geomorfológicos como planicies, colinas y montañas son producto de la interacción de un conjunto de ríos y la tectónica de la región (falla de La Victoria en sentido oeste-este) (Figura 1). Las pendientes son abruptas en las zonas montañosas alcanzando el $70 \%$ y en la depresión, la pendiente oscila entre 1 y $5 \%$ (INE, 2011).

El área de estudio presenta un clima Subhúmedo seco con precipitaciones anuales que oscilan entre los $921 \mathrm{~mm}$ y los $1063 \mathrm{~mm}$. El régimen de lluvias describe una distribución de tipo Unimodal con dos periodos definidos; una temporada lluviosa entre mayo a octubre, y una temporada seca entre noviembre y abril. las condiciones de altitud de la Depresión del Lago de Valencia (400-500 msnm) y su localización entre dos serranías, generan condiciones no tan frescas considerando el gradiente altotér- 
mico, más bien se mantienen con una disminución gradual lenta, y en general un poco cálida, regis- trándose en Valencia (478 $\mathrm{msnm})$, una temperatura media anual de $24,6{ }^{\circ} \mathrm{C}$ (Hernández et al., 2017).

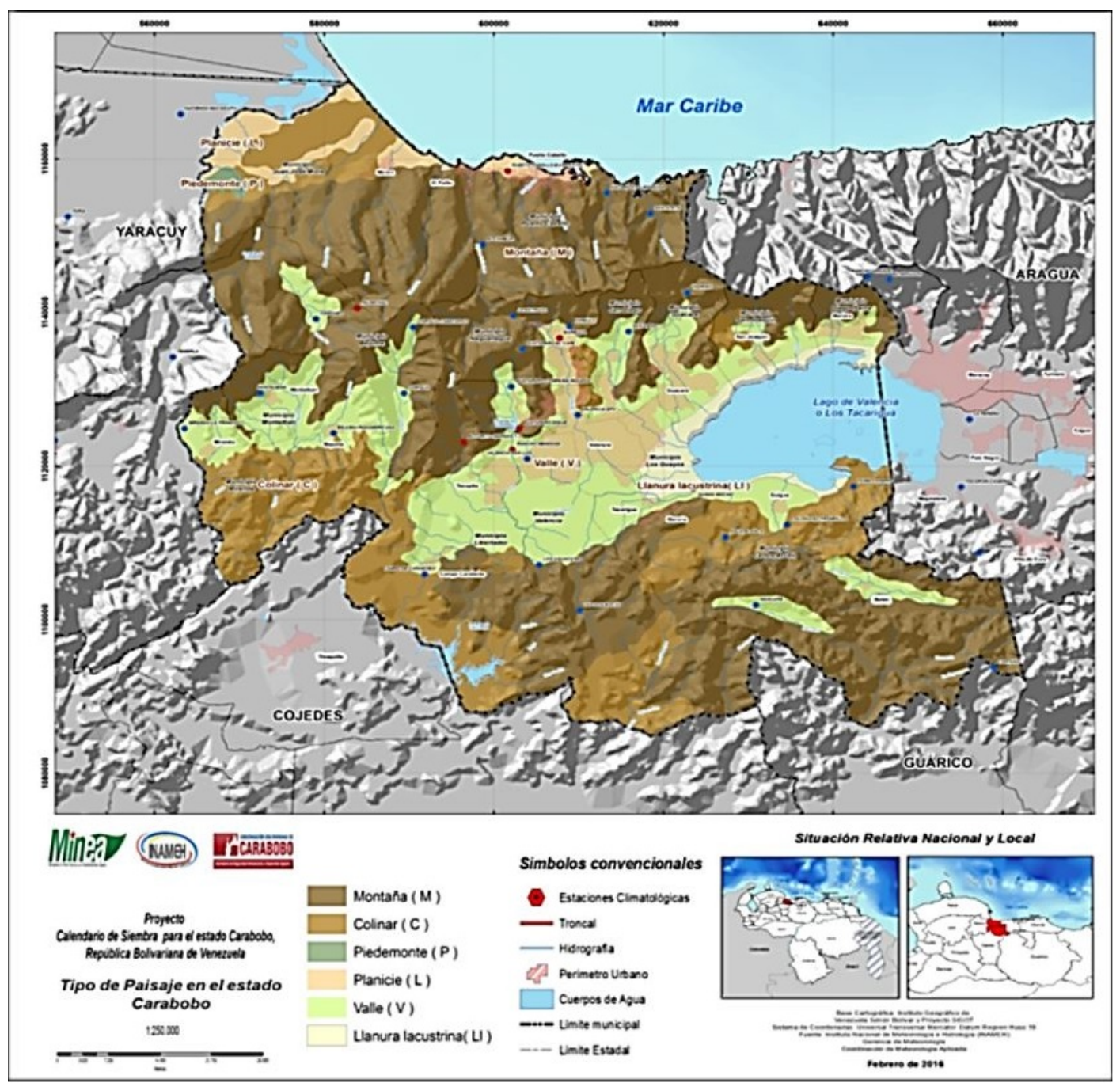

Figura 1. Tipo de Paisaje en el estado Carabobo, Venezuela (Hernández et al., 2017).

\subsection{Datos de precipitación}

Para este estudio se utilizaron los datos mensuales de precipitación (1969-1999) provenientes de ocho estaciones meteorológicas, ubicadas en la cuenca del Lago de Valencia, estado Carabobo (Tabla 1), los mismos fueron obtenidos del banco de datos de la Gerencia de Procesamiento y Calidad de datos del Instituto Nacional de Meteorología e Hidrología de Venezuela.

La selección de este periodo de tiempo (19691999) se basó en la exactitud de las series que con- sistió en el carácter correcto de los datos, así como la validez referida a la aplicabilidad de los datos con la finalidad de establecer las tendencias de las series temporales. La decisión de no considerar el periodo actual de datos de precipitación estuvo asociada a que más del 33\% del total de las estaciones climatológicas presentaban periodos ininterrumpidos y extensos de datos faltantes dentro del registro histórico, adicionalmente existió una porción importante de estaciones que fueron desmanteladas en el transcurso de 2001-2010, por lo tanto no contaban con datos de lluvia a partir del año 2000. 
Tabla 1. Descripción geográfica de las estaciones pluviométricas ubicadas en la cuenca del Lago de Valencia, estado Carabobo, Venezuela

\begin{tabular}{cccccc}
\hline Estación & Municipio & Paisaje & Norte & Este & Altitud (m.s.n.m) \\
\hline Vigirima & Guacara & Montaña & 1142558 & 622845 & 557 \\
San Diego & San Diego & Valle & 1137558 & 615956 & 460 \\
Valencia-GFV & Valencia & Valle & 1126726 & 609967 & 460 \\
Yuma-Caserio & Carlos Arvelo & Colinar & 1117317 & 642415 & 460 \\
Colonia El Trompillo & Carlos Arvelo & Colinar & 1112434 & 634579 & 450 \\
Agua Blanca & Carlos Arvelo & Colinar & 1110749 & 627339 & 515 \\
Las Dos Bocas & Valencia & Montaña & 1101292 & 610166 & 550 \\
Bárbula & Naguanagua & Montaña & 1136672 & 607806 & 493 \\
\hline
\end{tabular}

Se realizó un control de calidad a los datos de precipitación mensual para definir la proporción de datos faltantes, identificar valores fuera de lo normal y observar el entendimiento básico de la distribución de las series (Parra y Cortez, 2005; Olivares et al., 2013; Guevara, 2003); utilizando los programas JMP v.6 (Jones y Sall, 2011), InfoStat v.11 (Di Rienzo et al., 2011) y Vesper v.1.6 (Minasny, McBratney y Whelan, 2002).

\subsection{Análisis de las series temporales}

La metodología propuesta se inserta dentro de los procedimientos estadísticos propios del análisis de series temporales, el cual comprende tres fases de estudio: la primera relacionada con el cálculo de estadísticos descriptivos básicos (media, mediana, varianza, desviación estándar, coeficiente de variación, curtosis, asimetría, valores máximos y mínimos; el 1er y 3er cuartil); la segunda fase fue describir la evolución secular de la precipitación (19691999), la cual consistió en la tipificación de los valores anuales de precipitación y a partir de estos, ejecutar tanto el cálculo de medias móviles centradas en dos años, como el de la recta de tendencia calculada con el número mínimo de cuadrados; por último, el cálculo del rango percentil P90\%-P10\% como indicador de la variación de la amplitud de la serie.

\section{Resultados y discusión}

\subsection{Comportamiento de la precipitación mediana (1969-1999)}

La Figura 2 muestra el comportamiento de la mediana de precipitación para las estaciones bajo es- tudio. A lo largo y ancho de la cuenca del Lago de Valencia la cantidad de precipitación es sumamente variable tanto en el tiempo como en el espacio. Durante los meses de la época seca (enero a marzo) todas las localidades presentan precipitación mediana inferior a los $40 \mathrm{~mm}$, con una alta proporción de valores cero.

Durante los meses de abril y mayo se incrementan los valores de precipitación mediana, con un ascenso de noroeste a sureste, que indica el avance de la Zona de Convergencia Intertropical (ZCIT), conservándose valores en el rango $(50-150 \mathrm{~mm})$ para la mayoría de las localidades, excepto en Agua Blanca (Figura 2g) y Las Dos Bocas (Figura 2h) donde la mediana supera este rango.

Por otra parte, para los meses junio, julio y agosto ya está establecida la época lluviosa en la región de estudio, con valores de mediana que superan los $150 \mathrm{~mm}$ en la mayoría de las estaciones. La localidad de Yuma Caserío (Figura 2e) presenta valores inferiores a $150 \mathrm{~mm}$, siendo la localidad con valores más bajos en la época lluviosa. La localidad Las Dos Bocas presenta montos de mediana en el rango (180-240 mm) por estar ubicada en un clima ligeramente húmedo según Hernández et al. (2017).

Con respecto a los meses septiembre y octubre, estos presentan valores de mediana inferiores a 150 $\mathrm{mm}$, desarrollándose el patrón que comenzó a manifestarse en agosto, hacia el sur del estado la precipitación aumenta significativamente durante estos meses. En noviembre y diciembre se presenta en toda la zona de estudio un patrón de disminución de la lluvia. 


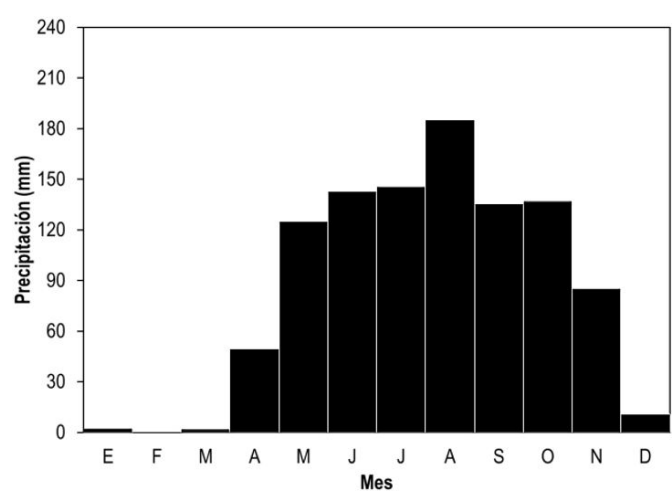

(a) Vigirima

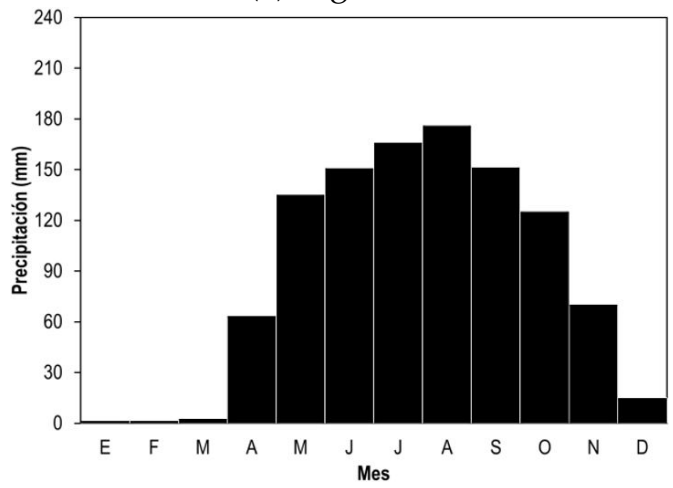

(c) Bárbula

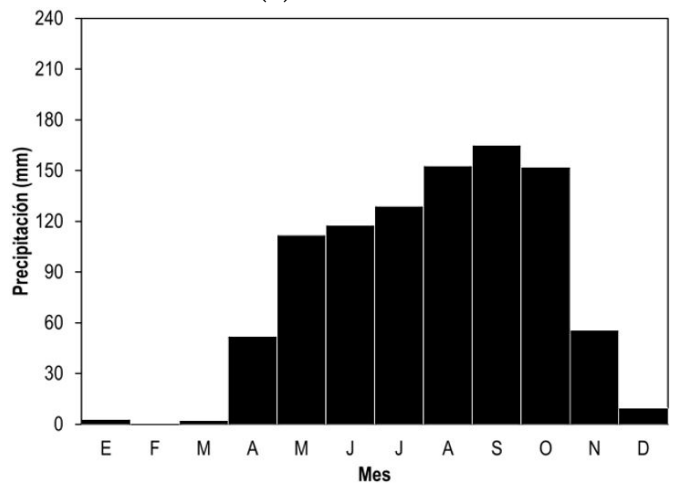

(e) Yuma Caserío

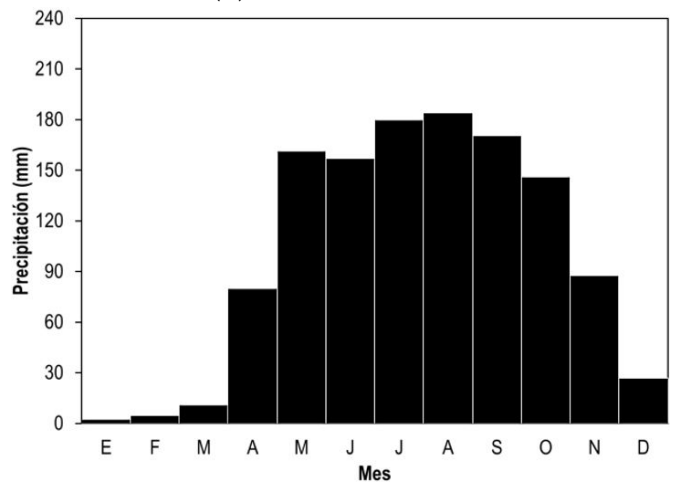

(g) Agua Blanca

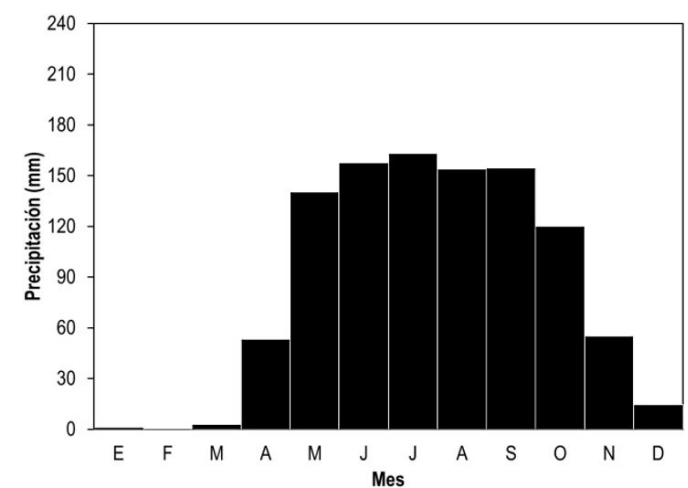

(b) San Diego

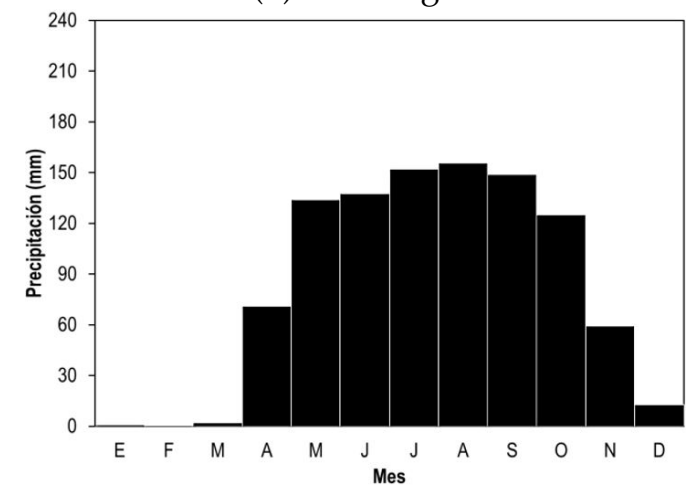

(d) Valencia GFV

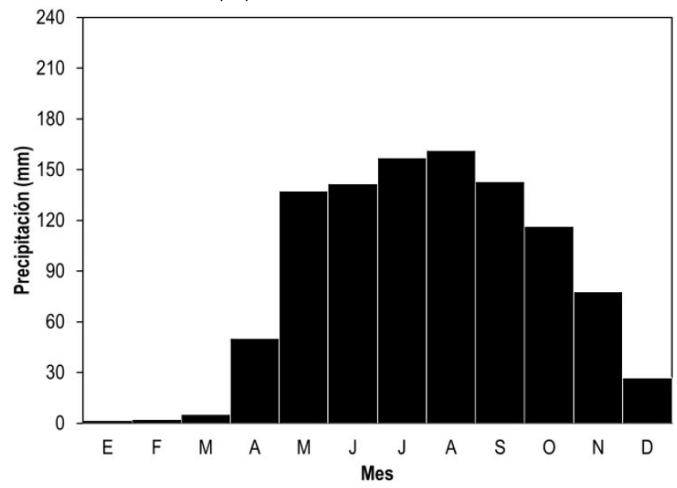

(f) Colonia El Trompillo

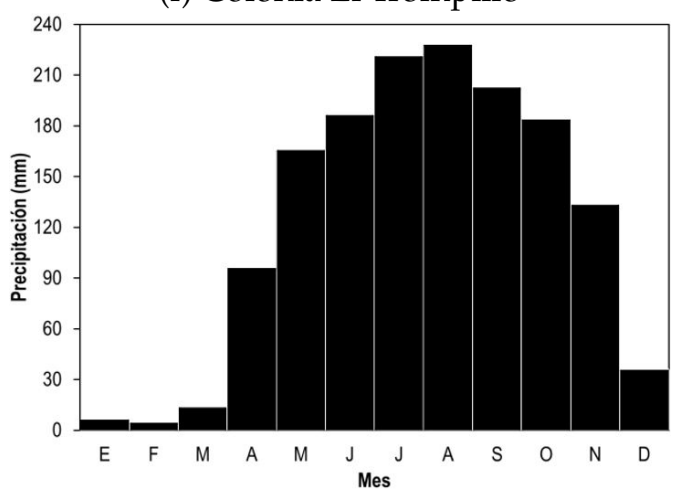

(h) Las Dos Bocas

Figura 2. Precipitación mediana (mm) para el periodo (1969-1999) en las estaciones bajo estudio: (a) Vigirima; (b) San Diego; (c) Bárbula; (d) Valencia GFV; (e) Yuma Caserío; (f) Colonia El Trompillo; (g) Agua Blanca; (h) Las Dos Bocas.

LA GRANJA:Revista de Ciencias de la Vida 27(1) 2018:86-102.

(C)2018, Universidad Politécnica Salesiana, Ecuador. 


\subsection{Comportamiento de los estadísticos descriptivos}

Las representaciones gráficas de cada localidad (Figura 3) mostraron valores aceptables y lógicos dentro de los rangos reportados por la Primera Comunicación Nacional de Cambio Climático en Venezuela (MARN, 2005). Una característica de esta región es la marcada estacionalidad de la precipitación, debida a la acción alternada de la zona de convergencia intertropical. El periodo lluvioso concentra más del $85 \%$ del total anual de precipitación, mientras que en los meses secos llueve muy poco siendo la precipitación mensual inferior a la evapotranspiración de referencia; es muy frecuente que en los meses de febrero y marzo la lluvia registrada sea de cero milímetros (Martelo, 2003).

Los resultados estadísticos que describen la estacionalidad de la lluvia en la zona bajo estudio coinciden con las características climáticas de la región (Guenni, Degryze y Alvarado, 2008; Olivares et al., 2013 , 2017). La variabilidad anual de la precipitación en Venezuela está altamente influenciada por las condiciones geográficas dominantes tales como la presencia de la cordillera de La Costa y su interacción con el mar Caribe; así como la migración de la Zona de Convergencia Intertropical que origina el régimen unimodal en la zona.

Los eventos generalizados secos de carácter relevante ocurren principalmente en meses de la época lluviosa y en los meses de noviembre y diciembre, trayendo consecuencias negativas para el crecimiento y desarrollo de los cultivos. Se evidencia una

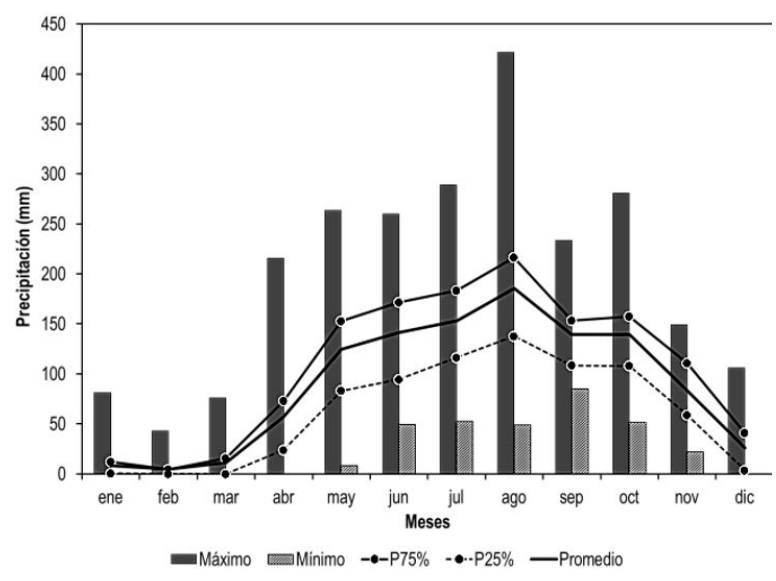

(a) diferencia significativa respecto a los eventos secos que se concentran en los meses de abril a agosto; al parecer los eventos lluviosos pueden ocurrir en cualquier momento debido al origen de la precipitación en la zona. Al respecto, Martelo (2003) señala que los eventos lluviosos registrados en las series históricas entre los meses de enero y febrero pueden ser con gran probabilidad la consecuencia de la llegada de Frentes Fríos, mientras que los eventos secos en la temporada lluviosa pueden deberse a un pasaje de Ondas del Este más alto de lo normal o a una temporada de huracanes especialmente intensa.

Una característica típica de la lluvia tropical, es la ocurrencia de gran intensidad; tal y como lo señalan González y Córdova (1992) con duraciones entre 30 minutos y 6 horas; a su vez, el estudio desarrollado por Goldbrunner (1984) establece que el 95\% de las precipitaciones caen en forma de chaparrones de 15 a 30 minutos de duración, frecuentemente en la tarde.

La zona de estudio se destaca por su producción agrícola y pecuaria especialmente en los valles occidentales y en las riberas del Lago de Valencia. Es principal productor de rubros como maíz, tabaco, algodón, café, cacao, caña de azúcar, cambur, plátano, ñame, papa y cítricos. En este orden de ideas, uno de los principales problemas que enfrenta la agricultura de secano es la gran variabilidad que presenta la lluvia espacial y temporalmente, lo cual repercute en la variabilidad del inicio, fin y longitud de la estación de crecimiento que son parámetros importantes dentro de la actividad agrícola.

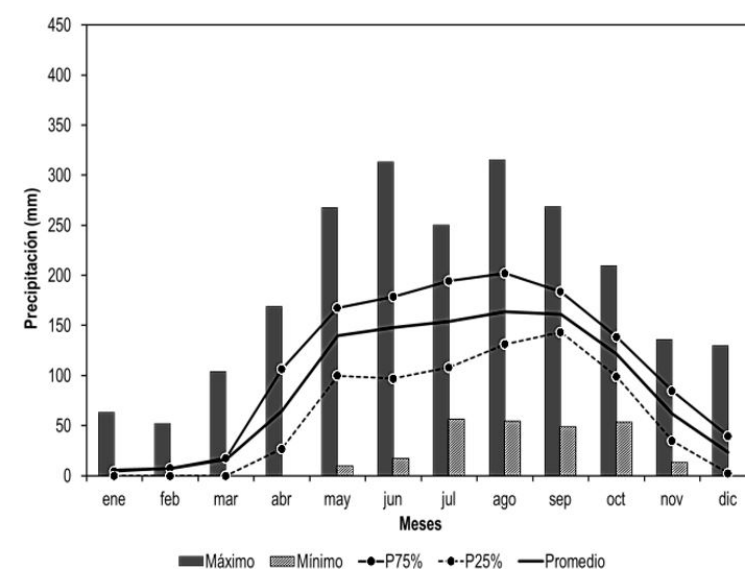

(b) 


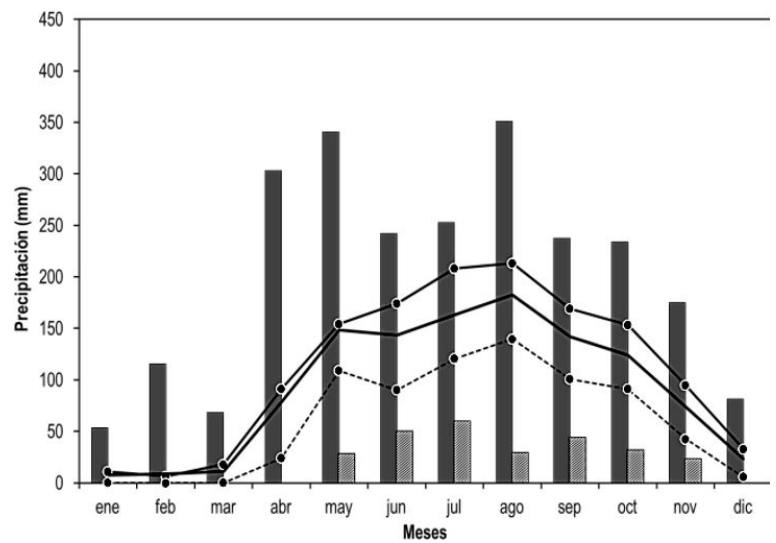

(c)

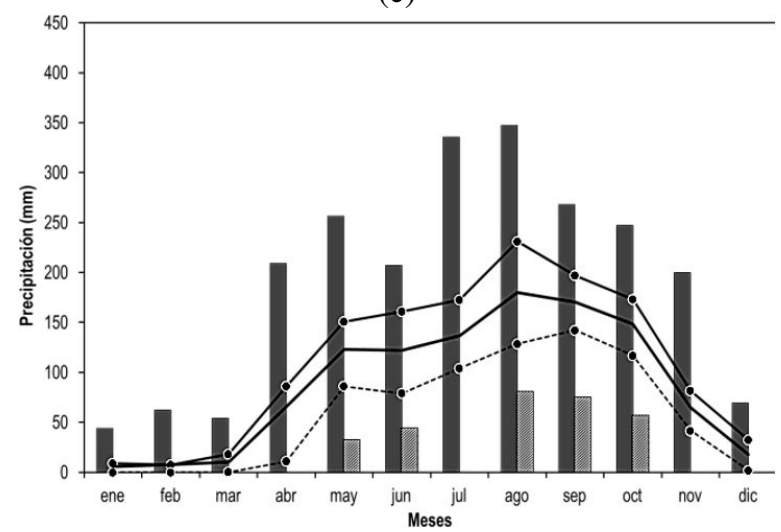

(e)

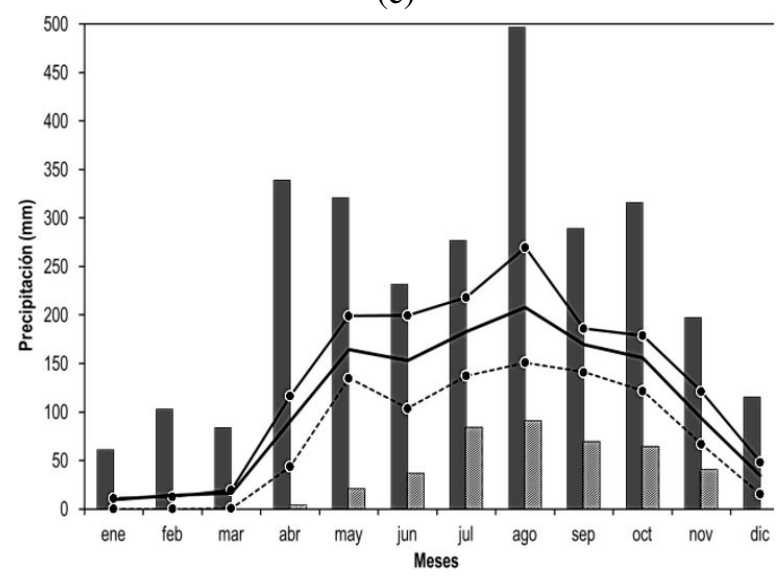

(g)

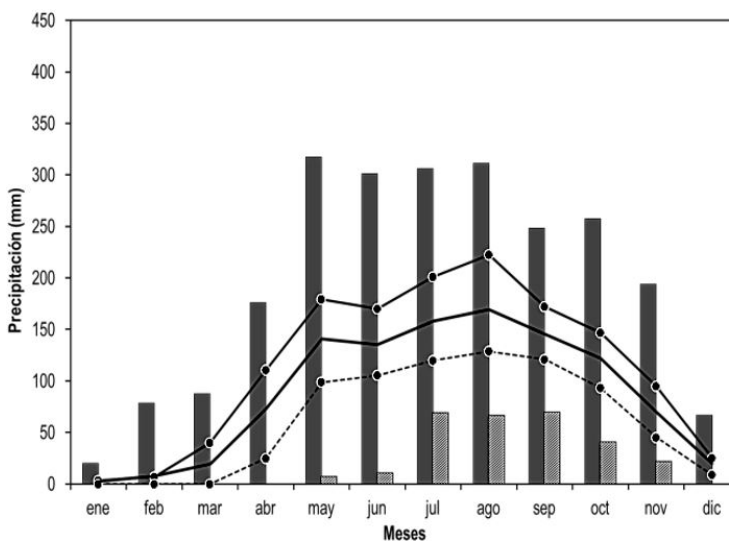

(d)

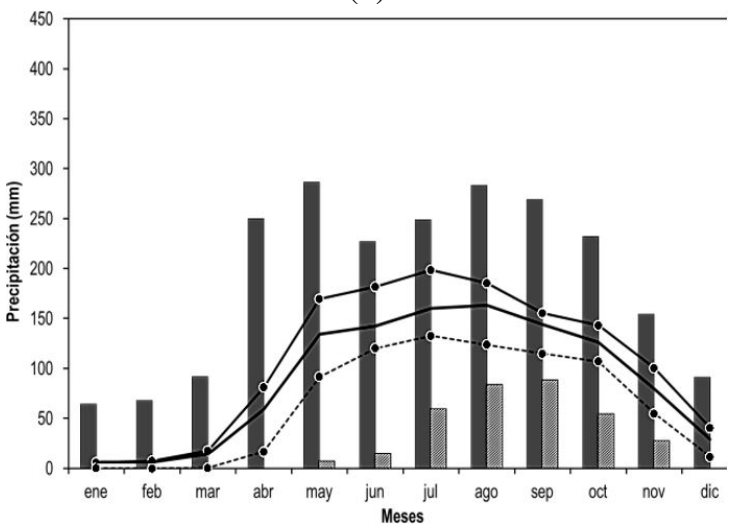

(f)

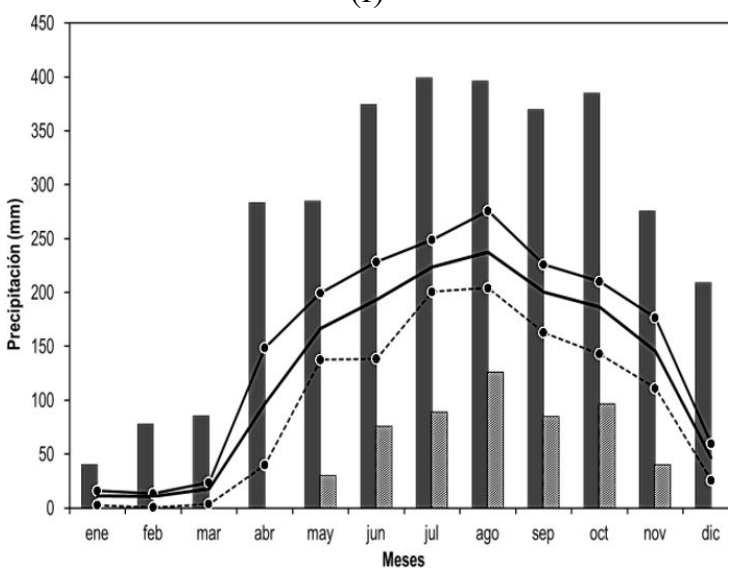

(h)

Figura 3. Comportamiento de la precipitación promedio mensual (mm), valores máximos, mínimos, percentiles $75 \%$ y $25 \%$ para las estaciones bajo estudio: (a) Vigirima; (b) San Diego; (c) Barbula; (d) Valencia GFV; (e) Yuma Caserio; (f) Colonia El Trompillo; (g) Agua Blanca; (h) Las Dos Bocas. 
En estas localidades donde la variabilidad de precipitación es elevada, no se recomienda utilizar el valor promedio, particularmente por estar ubicadas en una región subhúmeda, zonas donde suele utilizarse la captación de lluvia y donde la variabilidad de precipitación tiende a ser alta. Es más adecuado utilizar un valor cuya probabilidad de ocurrir sea mayor que el $50 \%$ (por ejemplo, $75 \%$ o más), dependiendo de las características locales de la precipitación, el tipo de uso y la situación de escasez. Generalmente, cuanto más severa es la situación de escasez y prioritario es el tipo de uso, menos incierta debe ser la cantidad de agua disponible.

El manejo de la información sobre el déficit hídrico vegetal puede orientar y hasta modificar las fechas de siembra de los cultivos con la finalidad de tener menor déficit de agua. Aunque pueda parecer obvio, este tipo de estudios permiten orientar al productor o planificador al momento de seleccionar las fechas de siembra, buscando coincidir los períodos de menor probabilidad de déficit (mayores valores de la lluvia de diseño) con el período más crítico de susceptibilidad de los cultivos, generalmente el reproductivo. Se trata de buscar un ajuste entre la demanda hídrica de los cultivos con la disponibilidad de agua en el periodo.

En particular, la longitud del ciclo de los cultivos que crecen en la zona de estudio (cereales, hortalizas y tubérculos) deberán ser más cortos que la longitud de la estación de crecimiento más probable (inferior a cinco meses de duración).

La falta de agua significativa, en un mes lluvioso y bajo condiciones de agricultura de secano, pone en apuros a los productores agrícolas de la zo- na, quienes frente a una realidad que se repite año tras año, deben elaborar una serie de estrategias para manejar con éxito esta situación. Considerando la magnitud del déficit hídrico en estas zona agrícolas del estado Carabobo, se debería enfrentar los episodios de sequía mediante una mayor dotación de recursos para la construcción de pozos profundos, aprovechando además causes de los ríos y quebradas cercanos.

Por el contrario, los excesos de agua, también ejercen una influencia considerable en la logística de la cosecha agrícola, por lo que, para aquellas zonas de amplio tamaño y cuyo paquete tecnológico lo permita, las maquinarias deberán estar en condiciones operativas para no perder tiempo de días de cosecha por roturas previsibles. Además, es probable que la red de caminos o carreteras agrícolas esté en una situación de intransitabilidad, por lo que se deberá organizar con tiempo el almacenaje en el campo y su posterior cuidado de acuerdo a la humedad de almacenaje y condiciones de almacenamiento.

\subsection{Evolución de la precipitación anual (1969-1999)}

Las curvas de las medias móviles (Figura 4), registran los valores más bajos en los años 1979 (sequia generalizada), 1985 y 1990 para las localidades San Diego (Figura 4b) y Bárbula (Figura 4c), coincidiendo con los mínimos valores de Vigirima (Figura 4a), a excepción del periodo 1994. Por su parte en Valencia (Figura 4d), la media móvil más baja se presentó durante los años 1997 y 1990.

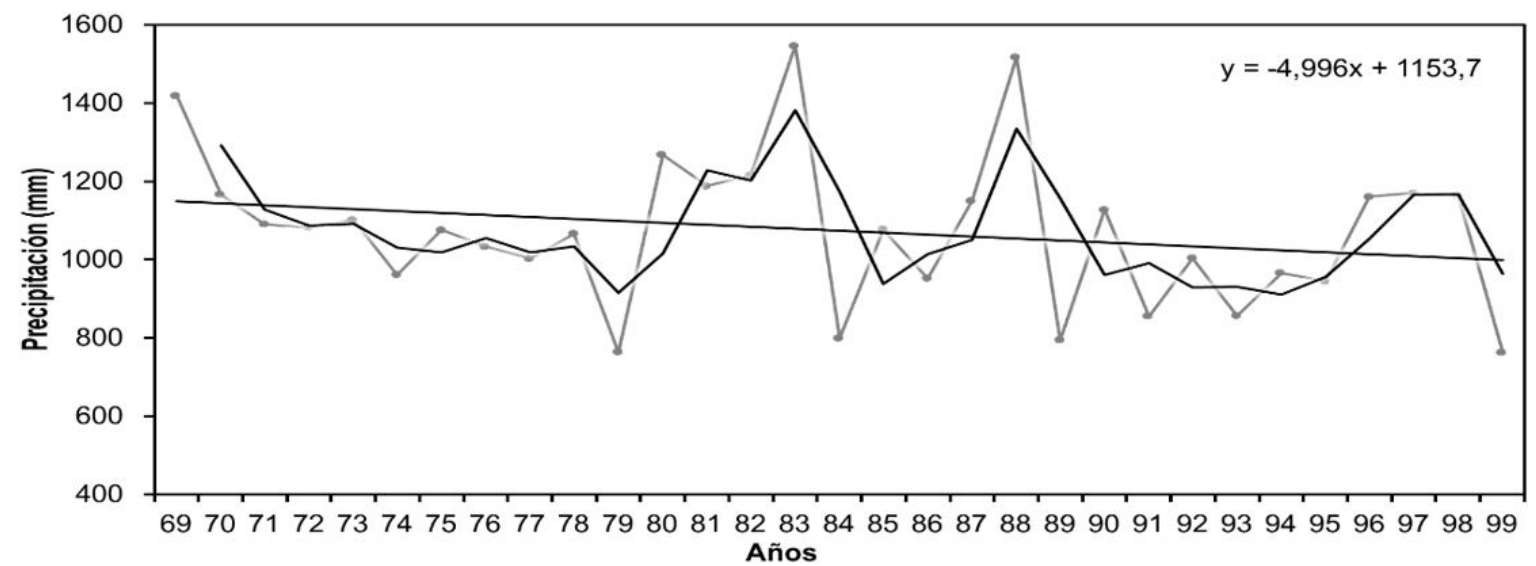

(a) 


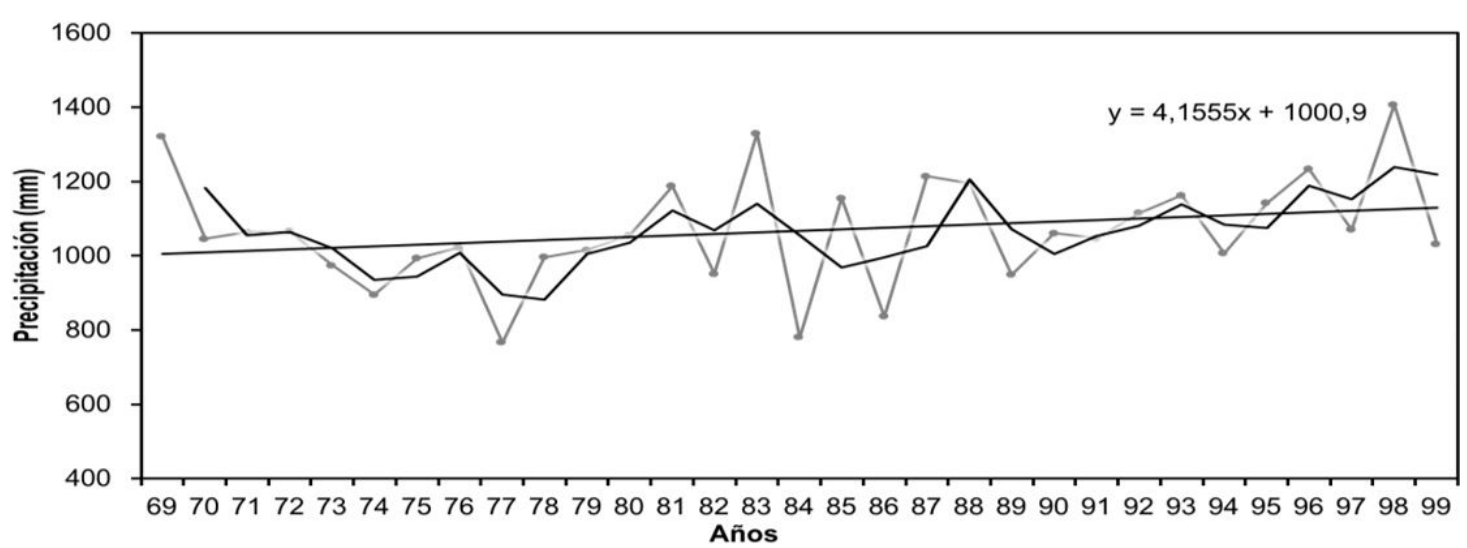

(b)

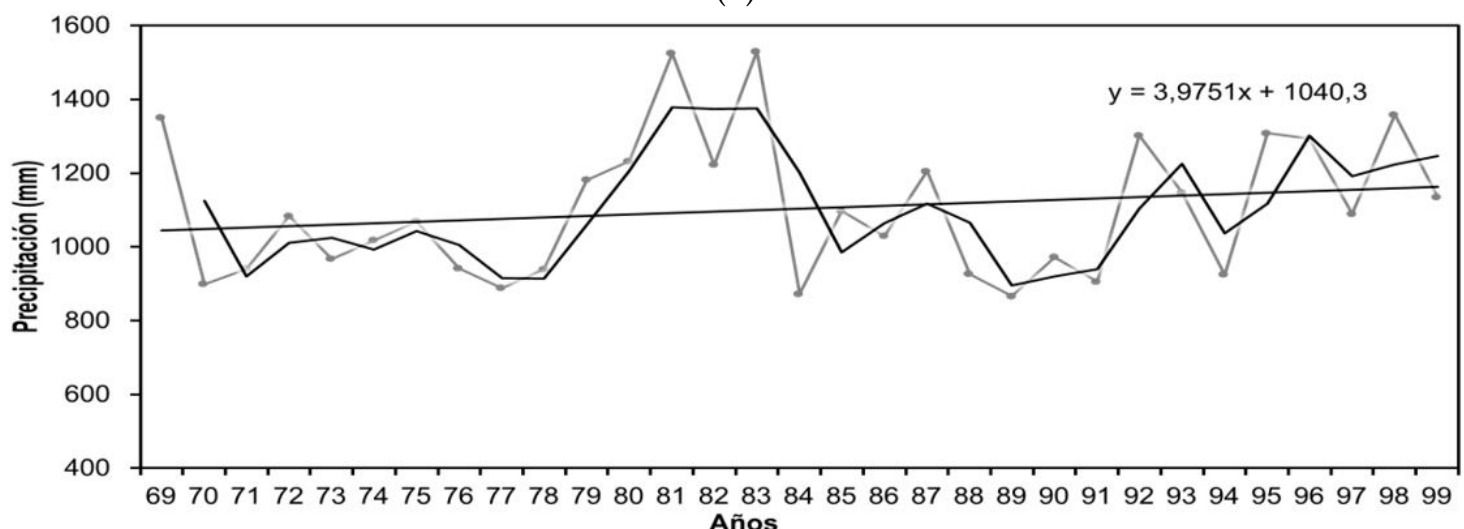

(c)

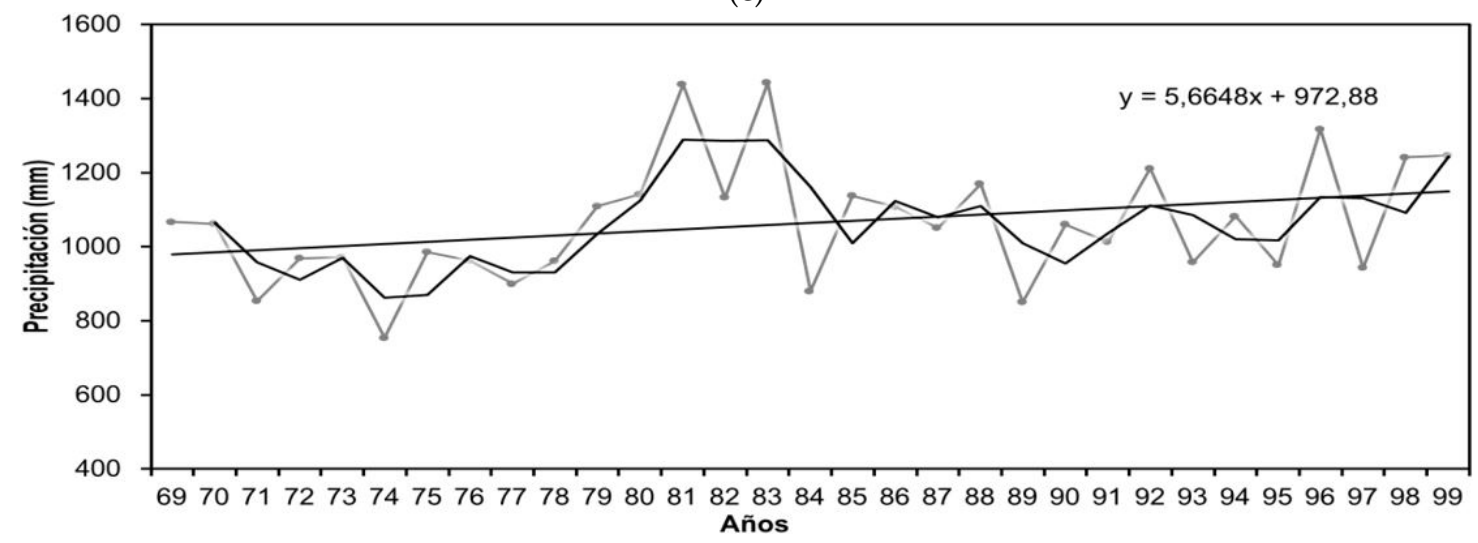

(d)

$\rightarrow$ Precipitación anual $\quad-2$ per. media móvil (Precipitación anual) -Lineal (Precipitación anual)

Figura 4. Evolución de las precipitaciones anuales (1969-1999) para las estaciones bajo estudio: (a) Vigirima; (b) San Diego; (c) Bárbula; (d) Valencia GFV.

De esta gráfica se desprende que la parte sureste del estado Carabobo presenta una tendencia negativa en la precipitación, representadas por las localidades Yuma Caserío (Figura 5a), Colonia El Trom- pillo (Figura 5b), cuyos valores más bajos de media móvil se registran durante los años 1977 y 1994; por su parte, en Agua Blanca (Figura 5c) los años más secos fueron 1974 y 1997, mientras que en Las Dos 
Bocas (Figura 5d) los años secos lo constituyen 1980 y 1989.

Para la mayoría de las localidades analizadas, el componente de tendencia no resulta estadísticamente significativo, sin embrago, los valores obtenidos de pendientes negativas para la zona de estudio están de acuerdo, al menos en signo, con las reportes de Guenni, Degryze y Alvarado (2008). Las pendientes negativas también pueden ser una evidencia de una mayor actividad del fenómeno ENSO (El Niño-Southern Oscilation) en años recientes, dado el impacto negativo de la fase caliente de ENSO (El Niño) sobre la precipitación de la región norte de Suramérica (Poveda, Waylen y Pulwarty, 2006).
El análisis de estas tendencias negativas, podría asomar la posibilidad de una reducción en el número de meses húmedos lo que limita el uso de cultivos tanto anuales como perennes, dependiendo de la magnitud de la reducción. La distribución de la precipitación se verá afectada, ejemplo de ello es el análisis de la distribución derivada del estudio de Martelo (2003), donde la mayor incidencia es en el mes de mayo época que corresponde a la preparación de las tierras y siembra de los principales cultivos en la mayoría de las zonas agrícolas; con incidencias también en el mes de agosto, mes que se corresponde con la floración, fructificación y llenado de granos principalmente de cereales.

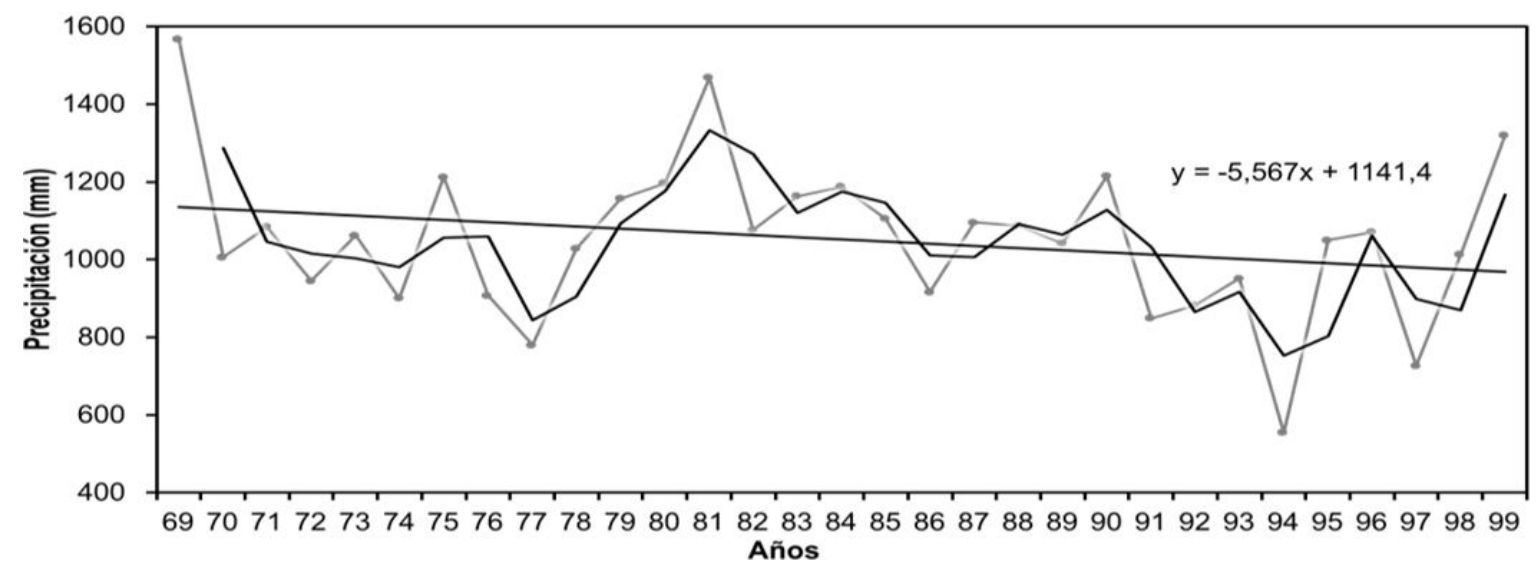

(a)

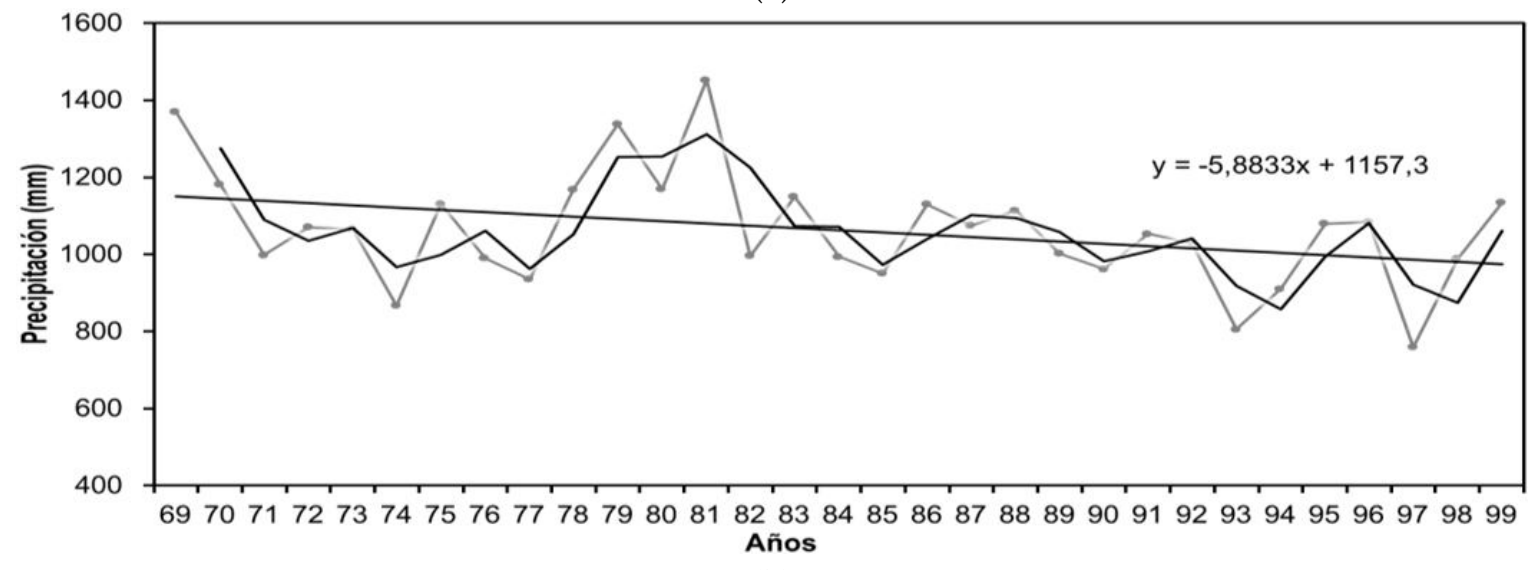

(b) 


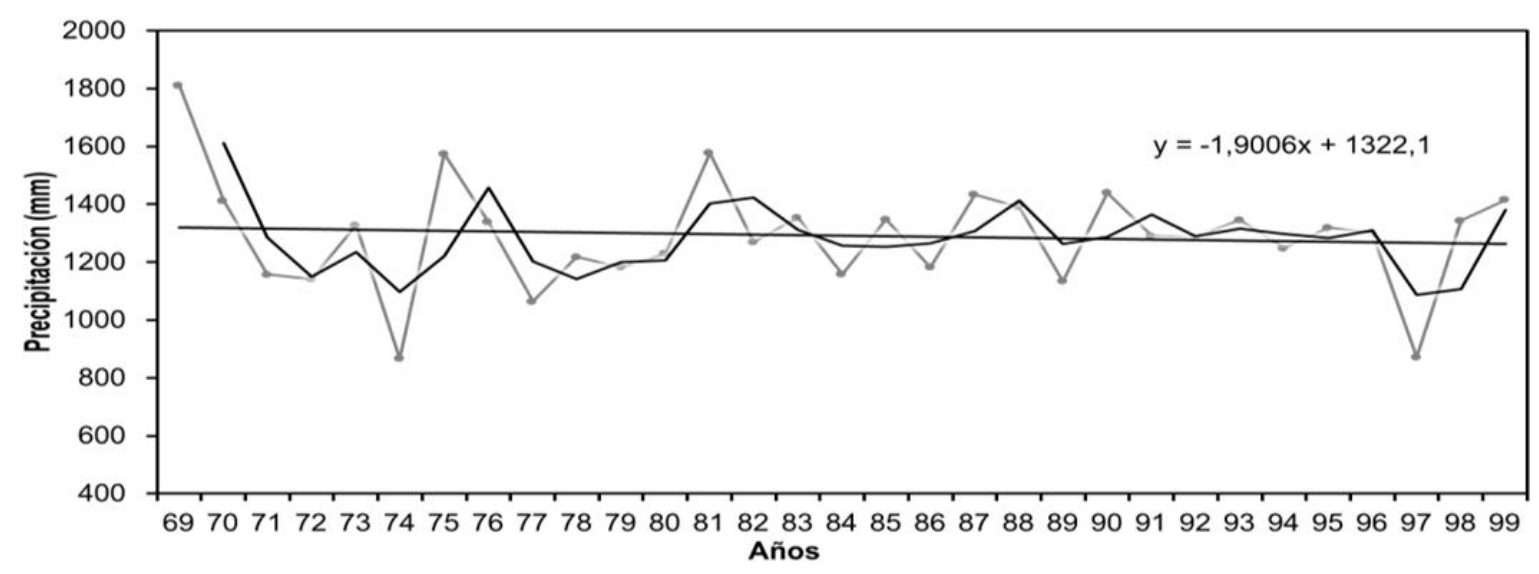

(c)

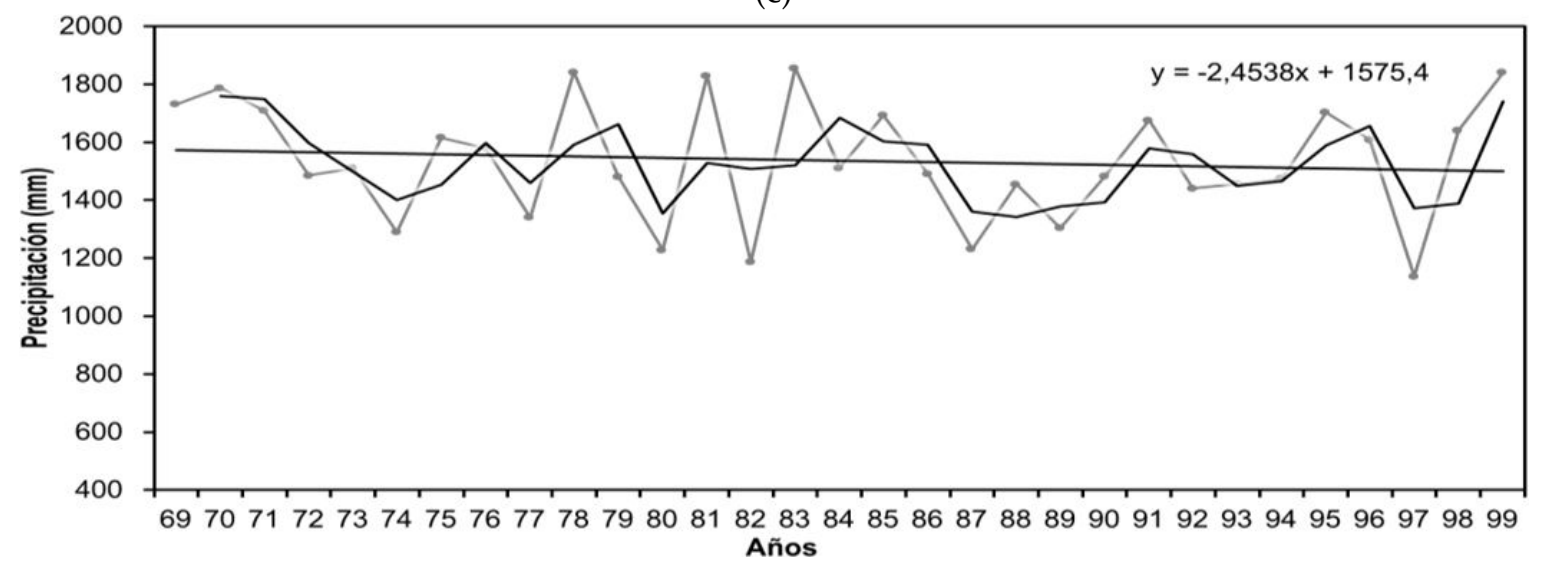

(d)

$\rightarrow$ Precipitación anual $\quad-2$ per. media móvil (Precipitación anual) -Lineal (Precipitación anual)

Figura 5. Evolución de las precipitaciones anuales (1969-1999) para las estaciones bajo estudio: (a) Yuma Caserío; (b) Colonia El Trompillo; (c) Agua Blanca; (d) Las Dos Bocas.

Este patrón, coincide con las amenazas asociadas al Cambio Climático, dado que estudios globales, han estimado entre otras, alteraciones en el sistema climático y cambios en la estacionalidad de la precipitación con un incremento en la intensidad de los periodos secos con fuertes repercusiones sobre el ciclo hidrológico (MARN, 2005; Ovalles et al., 2008).

De acuerdo a lo anterior, el estado Carabobo, presenta una superficie cosechada de estos rubros de 6.970 ha donde el $65 \%$ corresponde a la caña de azúcar, el $16 \%$ a los cereales, el $19 \%$ restante distribuido entre cacao, plátano, cambur, tabaco, pimentón y tomate. Ya en estas zonas la mayor parte de los rubros mencionados (cacao, caña, hortalizas, Musáceas y semilla de cereales) están al menos parcialmente bajo riego. Ello creara mayor competencia por el agua con otros usos como el urbano y exigi- rá métodos de riego mucho más eficientes que los actuales para mantenerse en la zona.

Ante el impacto estimado de reducción promedio de la oferta hídrica anual en las localidades Yuma Caserío, Colonia El Trompillo, Agua Blanca y Las Dos Bocas, se debe vislumbrar que es necesario el entendimiento de las posibles repercusiones por cambios en la concentración de la precipitación a escala diaria y horaria, que a su vez contribuirán a determinar de manera más precisa las adaptaciones futuras en los sistemas agroproductivos de Carabobo para hacer frente a los riesgos por cambios en la frecuencia e intensidad de eventos de la precipitación.

Es por ello que los impactos de las tendencias climáticas determinan la relevancia de emprender acciones coordinadas sobre el territorio agrícola, da- 
do que los cambios en las partes altas de las subcuencas que la conforman, afectarán también las actividades en zonas media y baja, así mismo en la población y los diferentes sectores agroproductivos.

Los resultados inducen un posible aumento en la precipitación mensual a menor altitud, sobre el Valle representativo en las localidades San Diego y Valencia, esto deberá implicar adaptaciones tecnológicas de algunas unidades de producción agrícola, principalmente para la agricultura que se desarrolla activamente en esta zona de la cuenca del Lago de Valencia, referidas por ejemplo a modificaciones sobre las fechas de siembra y la programación del riego. Sin embargo, los mayores impactos estimados de reducción en la precipitación anual hacia la zona sur, pueden significar fuertes repercusiones sobre un descenso en la oferta hídrica y las consecuencias en menos agua para satisfacer las necesidades hídricas de los cultivos.

\subsection{Análisis del rango percentil $\mathbf{P 9 0 \% \text { - }}$ P10\%}

Este indicador proporciona información sobre la amplitud de la variación en la serie, donde el $87 \%$ de los valores de la serie presentan diferencias menores a este rango, y solo el $13 \%$ de los valores totales de todas las localidades bajo estudio son considerados como valores extremos. Esta información permitiría en un sentido muy amplio, identificar aquellos años donde la precipitación anual recibida comparada con la precipitación de la estación lluviosa (mayo a octubre) no logra ser significativa o suficiente para cubrir las demandas de agua de los cultivos, para el caso de extremos secos o por el contrario, que se generen condiciones de excesos de agua en años extremos húmedos, lo que hace el manejo del agroecosistema muy vulnerable.

Al graficar los montos de precipitación anual y estacional (mayo a octubre), en conjunto con la ubicación de los años particulares con relación a los percentiles obtenidos a partir de la serie histórica de datos, se definen los extremos húmedos y secos para dos localidades representativas en el área de estudio. Por una parte, en Vigirima (Figura 6a) se identi- fican los años 1983 y 1988 como extremos húmedos, es decir, estos años presentan montos anuales y estacionales por encima del percentil 90. La ocurrencia de estos eventos pueden determinar el fracaso de la cosecha, la disminución drástica de los rendimientos, la afectación de la plantación por condiciones propicias para el ataque de plagas y aparición de enfermedades, así como el aumento del riesgo climático que puede afectar la estabilidad de la agricultura en la zona. Por otra parte, los años 1979 y 1999 corresponden con los valores extremos secos (<800 mm), ubicándose por debajo de los percentil 10 de la lluvia anual y estacional.

Para el periodo analizado, los resultados descritos evidencian que el fenómeno ENOA (El NiñoOscilación Austral) puede explicar de forma parcial la ocurrencia de meses extremadamente secos o Extremos húmedos a escala local durante las temporadas húmedas y secas en la cuenca del lago de Valencia (Paredes et al., 2015; Olivares et al., 2016).

Según Cárdenas, García y Gil (2002), El Niño presenta una mayor incidencia sobre los extremos pluviométricos que La Niña a escala local y suele estar relacionado con condiciones anómalamente secas con moderada cobertura espacial. La moderada a escasa conexión entre las fases activas de ENOA identificada a escala local sugiere que otros factores no evaluados incidieron de forma determinante sobre ciertos eventos pluviométricos extremos (por ejemplo, la sequía generalizada de 1979 se dio durante la fase neutra de ENSO).

Las estrategias de manejo del agua en las localidades bajo estudio estarían orientadas a mantener infiltración elevada en el suelo; la prevención y control de la escorrentía, dado el riesgo alto de erosión hídrica en el periodo húmedo para zonas montañosas, además de promover técnicas para captar y almacenar agua en el periodo húmedo para uso en el periodo seco (Ovalles et al., 2008). Así mismo, está claro que el uso de técnicas de mejoramiento de agua en el suelo, aprovechamiento de la escorrentía o utilización del riego (dependiendo de la disponibilidad de agua) es muy importante para la producción agrícola de las localidades abordadas. También ayudaría el uso de cultivos adaptados al ecosistema local en función del tipo de clima y suelo. 


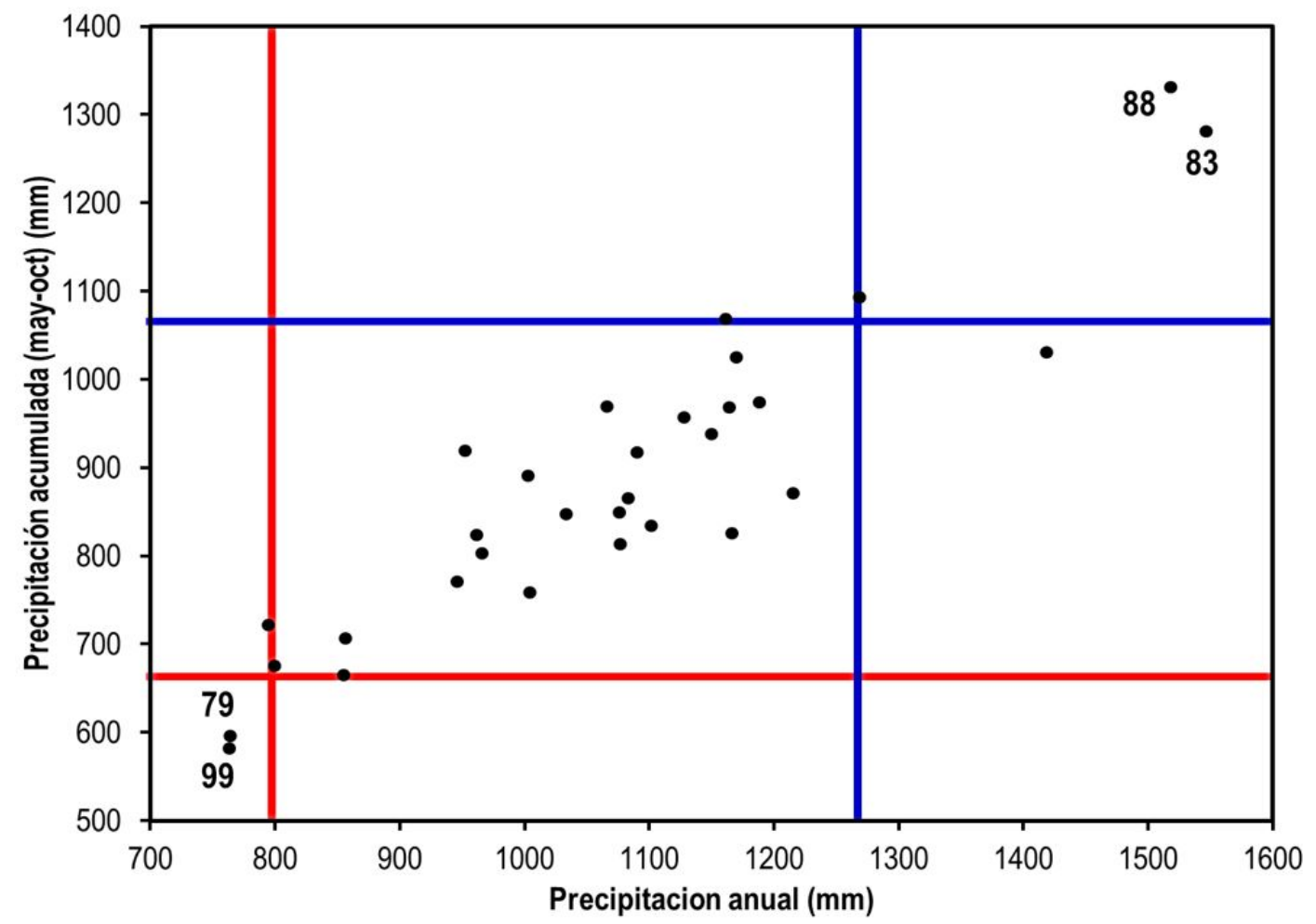

(a)

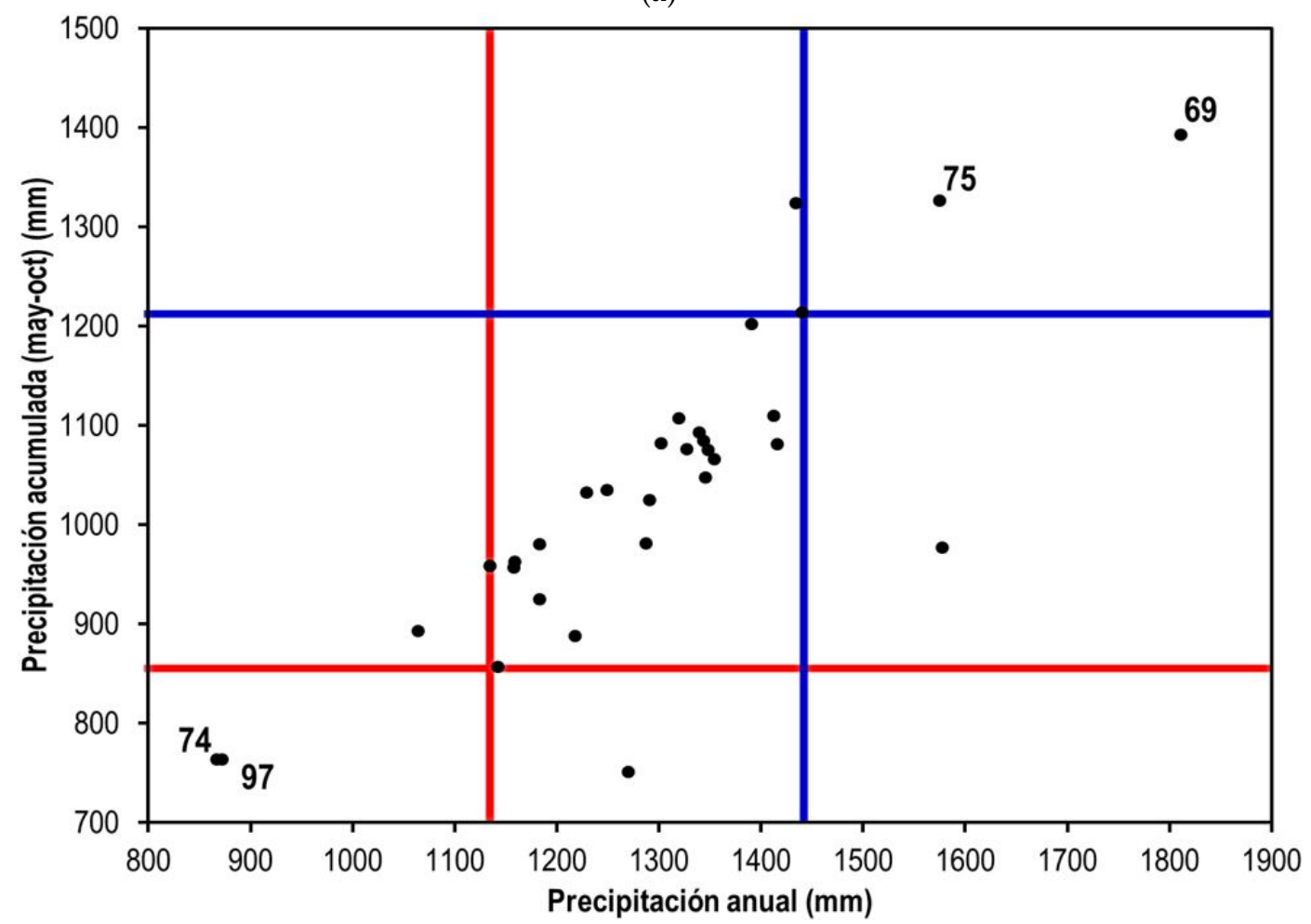

(b)

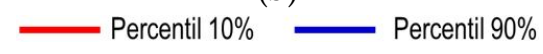

Figura 6. Rango percentil P90\%-P10\% de la precipitación anual y la precipitación acumulada entre la época lluviosa (mayo a octubre) en las localidades bajo estudio: (a) Vigirima; (b) Agua Blanca.

La Granja:Revista de Ciencias de la Vida 27(1) 2018:86-102.

(C)2018, Universidad Politécnica Salesiana, Ecuador. 
En la localidad de Agua Blanca (Figura 6b), se identificaron los años 1969 y 1975 como extremos húmedos, siendo los valores de lluvia anual y estacional superiores a los percentiles 90 de la serie, mientras que los años 1974 y 1997 son considerados como los extremos secos se la serie, ubicándose por debajo del percentil 10. En este caso, ambos valores de extremo seco son superiores a $800 \mathrm{~mm}$, lo que se interpreta que bajo estas condiciones, los extremos húmedos tendrían un mayor impacto que los extremos secos.

En función a lo descrito, es preciso que este tipo de análisis se realice con información diaria debido fundamentalmente a que para el caso de la agricultura de secano, la evaluación de estas situaciones extremas requiere de herramientas que incluyan la variabilidad interanual con enfoque de escala diaria con la firme convicción de obtener una mayor idea del riesgo climático en las zonas agrícolas.

\section{Conclusiones}

La variabilidad interanual de la precipitación es elevada, incluso en los meses más lluviosos. Así mismo, los análisis realizados evidencian como es de esperar, un aumento de la variabilidad en la época seca. Es de considerar que los resultados mostrados en este estudio tienen gran importancia desde el punto de vista del manejo sostenible del agua para la agricultura, ya que implica la necesidad de conocer en detalle local, cuales son los rangos principales de la precipitación que tienen efectos directos sobre los sistemas agro productivos, en especial, cuando este elemento climático es el más variable de todo el sistema.

Los resultados demostraron el comportamiento de la variabilidad estacional e interanual de la lluvia de modo general y su posible influencia en la agricultura de secano desarrollada en la cuenca del Lago de Valencia, Carabobo, con esto se obtiene un conocimiento básico y amplio sobre este elemento climático, lo cual permitirá una aproximación mucho más asertiva en la toma de decisiones estratégicas en territorios agrícolas.

La vocación agrícola en las tierras bajas de la cuenca del lago de Valencia, la hace muy vulnerable a los episodios secos persistentes, donde los cultivos de secano como sorgo industrial, maíz, hortalizas, frutas (musáceas) y cítricos merman sus rendimientos durante la ausencia prolongada de lluvias, lo que afecta al sector agrícola de la región.

\section{Agradecimiento}

Se agradece el apoyo financiero de la Secretaría de Seguridad Alimentaria y Desarrollo Agrario de la Gobernación del estado Carabobo, Venezuela y a la Gerencia de Meteorología Aplicada del Instituto Nacional de Meteorología e Hidrología (INAMEH).

\section{Referencias}

Cárdenas, P, L. F García y A. Gil. 2002. Impacto de los eventos El Niño-Oscilación del Sur en Venezuela. Corporación Andina de Fomento. [en línea] disponible en: https://goo.gl/HNHYr1.

Comerma, J y R. Parede. 1978. “Principales limitaciones y potencial agrícola de las tierras en Venezuela." Agronomía Tropical 28(2):71-85. [en línea] disponible en: https://goo.gl/phU4uG.

Cortez, A, M. F Rodríguez, J. C Rey, D Lobo, F Ovalles, D Gabriels y R. M. Parra. 2011. “Análisis de la agresividad y concentración de las precipitaciones en Venezuela. I. Región noroccidental." Bioagro 23(1):13-18. [en línea] disponible en: https://goo.gl/rEXu4Y.

Di Rienzo, J. A, F Casanoves, M. G Balzarini, L González, M Tablada y C. W. Robledo. 2011. InfoStat versión 2011. Grupo InfoStat, FCA. Universidad Nacional de Córdoba, Argentina. [en línea] disponible en: https://goo.gl/GzbBA5.

Goldbrunner, A. W. 1984. Atlas climatológico de Venezuela 1951-1980. Maracay: Servicio de Meteorología y Comunicaciones de la Fuerza Aérea Venezolana.

González, M y J. R. Córdova. 1992. Análisis estadístico de la precipitación máxima probable en Venezuela. In IV Encuentro Nacional de Clima Agua y Tierra. IV Jornadas Nacionales de Hidrología, Meteorología y Climatología. IX Jornadas de Riego Mejoramiento de Tierras, Caracas, Venezuela. [en línea] disponible en: https://goo.gl/hhFTTt.

Guenni, L, E Degryze y K. Alvarado. 2008. “Análisis de la tendencia y la estacionalidad de la precipitación mensual en Venezuela." Revista Colombiana de Estadística 31(1):41-65. [en línea] disponible en: https://goo.gl/vYemZe. 
Guevara, E, J. E Guevara y E. García. 2008. “Régimen climático y patrón espacial de las lluvias en la cuenca del Lago de Valencia, Venezuela." Revista de Climatología 8:29-40. [en línea] disponible en: https://goo.gl/mBwscQ.

Guevara, J. M. 2003. Métodos de estimación y ajuste de datos climáticos. Consejo de desarrollo científico y humanístico. UCV. [en línea] disponible en: https://goo.gl/LfLMWP.

Hernández, R, Y Pereira, J. C Molina, R Coelho, B Olivares y K. Rodríguez. 2017. Calendario de siembra para las zonas agrícolas del estado Carabobo en la República Bolivariana de Venezuela. Universidad Internacional de Andalucía, España. [en línea] disponible en: https://goo.gl/jrZJiM.

INE. 2011. Informe Geoambiental del Estado Carabobo. Instituto Nacional de Estadística, Venezuela. [en línea] disponible en: https://goo.gl/PKyDnT.

Jones, B y J. Sall. 2011. "JMP statistical discovery software." Wiley Interdisciplinary Reviews: Computational Statistics 3(3):188-194. [en línea] doi: http: //www.dx.doi.org/10.1002/wics.162.

Lobo, D, A Cortez, M. F Rodríguez, F Ovalles, J. C Rey, D Gabriels y R. M. Parra. 2010. "Análisis de la agresividad y concentración de las precipitaciones en Venezuela. I. Región de los llanos." Bioagro 22(3):169-176. [en línea] disponible en: https://goo.gl/EDH95z.

MARN. 2005. Primera Comunicación Nacional en Cambio Climático de Venezuela. Caracas: Programa de las Naciones Unidas para el Desarrollo (PNUD). Ministerio del Ambiente y de los Recursos Naturales, Venezuela. [en línea] disponible en: https: //goo.gl/qhQX6o.

Martelo, M. T. 2003. Metodología para la selección de Modelos de Circulación General de la Atmósfera y Escenarios Climáticos a incluir en la Primera Comunicación Nacional en Cambio Climático de Venezuela. Proyecto MARN-PNUD VEN/00/G31, Dirección de Hidrología y Meteorología, Ministerio del Ambiente y los Recursos Naturales. Caracas - Venezuela.

Minasny, B, A. B McBratney y B. M. Whelan. 2002. Vesper version 1.6. Australian Centre for Precision Agriculture. University of Sidney, Australia. [en línea] disponible en: https://goo.gl/s2HMX4.
Olivares, B, A Cortez, D Lobo, R Parra, J. C Rey y M. Rodríguez. 2016. "Estudio de la Sequía Meteorológica en Localidades de los Llanos de Venezuela Mediante el Índice de Precipitación Estandarizado." Revista Acta Nova 7(3):267-283. [en línea] disponible en: https://goo.gl/FCLiog.

Olivares, B, A Cortez, R Parra, M Rodríguez y E. Guevara. 2013. "Aplicación de procedimientos estadísticos para el control de calidad de las series de precipitación mensual de los llanos orientales venezolanos." Revista de la Facultad de Agronomía (LUZ) 30(3):367-391. [en línea] disponible en: https://goo.gl/meceBY.

Olivares, B, L Caraballo y J. Torrealba. 2013. "Variabilidad del régimen de precipitación en el período 1990-2009 en la localidad de El Tigre estado Anzoátegui, Venezuela." Revista de la Facultad de Agronomía (LUZ) (30):19-32. [en línea] disponible en: https://goo.gl/ViUrtG.

Olivares, B, M. L Zingaretti, J. A Demey Zambrano y J. R. Demey. 2017. "Aplicación del método STATIS-ACT al régimen de lluvias en la Región Oriental Venezolana. UNED." Cuadernos de investigación. UNED 9(1):97-106. [en línea] disponible en: https://goo.gl/PEFbFb.

Olivares, B. O. 2009. “Características de interés agrícola de la precipitación en la agricultura de secano." INIA Divulga (14):25-27, [en línea] disponible en: https://goo.gl/9pUfko.

Olivares, B, R Parra, E Guevara, A Cortez y M. F. Rodríguez. 2012. "Patrones de homogeneidad pluviométrica en estaciones climáticas del estado Anzoátegui, Venezuela." Multiciencias 12(Extraordinario):11-17. [en línea] disponible en: https:/ /goo.gl/RENec1.

OMM. 2009. La Organización Meterorológica Mundial da un vistazo. Fuentes Mixtas, Organización Meterorológica Mundial, [en línea] disponible en: https://goo.gl/fvaEQo.

Ovalles, F, A Cortez, M. F Rodríguez, J. C Rey y E. Cabrera-Bisbal. 2008. "Variación geográfica del impacto del cambio climático en el sector agrícola en Venezuela." Agronomía Tropical 58(1):37-40. [en línea] disponible en: https:/ /goo.gl/bR3EGv.

Paredes, F, E Guevara-Pérez, H Barbosa-Alves y C. Uzcátegui-Briceño. 2015. “Tendencia de la 
precipitación estacional e influencia de El NiñoOscilación Austral sobre la ocurrencia de extremos pluviométricos en la cuenca del lago." Tecnología y Ciencias de la Luz 6(6):33-48. [en línea] disponible en: https://goo.gl/G75Cc5.

Parra, R y A. Cortez. 2005. "Control de calidad de series de precipitación de las series de precipitación del INIA Venezuela en el periodo 19702000." Revista Argentina de Agrometeorología (56):63-73.

Poveda, G, P Waylen y R. Pulwarty. 2006. “Annual and Inter-annual Variability of Present Climate in Northern South America and Southern Mesoamerica." Palaeogeography, Palaeoclimatology, Palaeoecology 234(1):3-27. [en línea] doi: https:// doi.org/10.1016/j.palaeo.2005.10.031.
Pradere, R. 1999. "Definición de patrones homogéneos de pluviosidad en los llanos centrales venezolanos mediante Kriging factorial." Agronomía Tropical 49(3):297-325. [en línea] disponible en: https://goo.gl/RzB5Lg.

Toledo, V y D. Hernández-Szczurek. 2003. Estudio de la variabilidad temporal y espacial de las lluvias anuales de la depresión de Carora, estado Lara, Venezuela. In Evaluación de parámetros y procesos hidrológicos en el suelo. VII Escuela Latinoamericana de Física de Suelos. La Serena - Chile. [en línea] disponible en: https://goo.gl/wS6CHe.

Zinck, J. A. 2012. Geopedología. Elementos de Geomorfología para Estudios de Suelos y Riesgos Naturales. ITC Special Lecture Notes Series. [en línea] disponible en: https://goo.gl/5bKvFm. 